\title{
Evaluation of Different Levels of New Post Emergence Individual Herbicidal Mixture Over Reference Herbicide in Soybean [Glycine max (L.)]
}

\author{
Krishna Kumar*, Madhu Mali, Shrikant Chitale and Tarun \\ IGKV, Raipur, India \\ *Corresponding author
}

\section{Keywords}

Herbicides, Weed flora, Weed control, Soybean and Economics

Article Info

Accepted:

26 October 2018

Available Online:

10 November 2018

\section{A B S T R A C T}

A field experiment was conducted in 2016 at Indira Gandhi Krishi Vishwavidyalaya, Raipur, Chhattisgarh during kharif season. Bioefficacy of new BAS 835 UBH post emergence herbicide mixture in soybean [Glycine max (L.)]. A study evaluates the effect in soybean of early BAS 835 UBH post emergence mixture herbicide, against weeds. The dry weight, species wise and total density of weeds viz., Echinochloa colonum, Cynodon dactylon, Alternanthera sessilis, Cyperus rotundus, Euphorbia geniculata, Digitaria sanguinalis, Phyllanthus niruri, Eragrostis pilosa and Commelina benghalensis were maximum in untreated check whereas minimum at 20 and 40 DAS under hand weeding twice. Early post emergence application of alone herbicide imazathapyr 10\% SL @ $100 \mathrm{~g}$ a.i. ha ${ }^{-1}$ followed by imazamox $12 \%$ SL @ $42 \mathrm{~g}$ a.i.ha ${ }^{-1}$ reduced significantly the density and dry weight of all broad leaf, sedges, grassy weeds. Among the mixture herbicide applied, BAS 835UBH (imazethapyr $23 \mathrm{~g} / \mathrm{l}+$ imazamox $23 \mathrm{~g} / \mathrm{l}+$ bentazone $460 \mathrm{~g} / \mathrm{l}$ ) @ $800 \mathrm{~g}$ a.i.ha ${ }^{-1}$ gave significantly effective control the density and dry weight of weeds against other mixture herbicide treatment

\section{Introduction}

Soybean [Glycine $\max$ (L.) Merrill] has an important place in world's oilseed cultivation scenario due to its high productivity, profitability and vital contribution towards maintaining soil fertility. Soybean is the only crop which has been included both in the categories of oilseed and pulses. Currently, India ranks fourth in respect to soybean production in the world and soybean has established itself as a major rainy season crop in the rainfed agro-ecosystem of central and peninsular India.
Though the potential productivity of this crop is higher, realization into net profit is much lower. The reasons for low productivity are mainly non adoption of the proper package and practices, the major being insufficient weed control practices. Weeds compete in initial stages with crop for limited essential resources and seriously depress growth and development of crop (Singh and Kharwara, 1984). Adoptions of adaptable weed control measures improve the crop's productivity. There are various weed management practices available to manage weeds. Among them herbicides are found effective one. The 
practice of hand-weeding and mechanical weeding at the critical crop-weed competition stage is labourious and due to continuous rainfall it is difficult. Pre-emergence herbicides have some limitation like limited time span for application and scarcity of water for spraying. Therefore, the use of postemergence herbicides alone and also in mixture should be practiced. Hence use the post-emergence herbicides have better prospects.

In soybean, the weed flora as observed from the unweeded control plots consist of $58 \%$ sedges, 32\% broad-leaved and 10\% grasses (Kumar and Das, 2008). At early stage of soybean growth, the weed competition is critical. The post and pre-emergence herbicides are used by the farmers of the state to reduce the crop -weed competition, but there is always scope to work on newer herbicides and mixtures of herbicides for effective, timely and economical control of weeds for efficient utilization of applied inputs.

\section{Materials and Methods}

The experiment using RBD design, during kharif 2016 was conducted, at Indira Gandhi Krishi Vishwavidyalaya, Raipur situated at latitude of $21^{\circ} 25^{\prime} \mathrm{N}$, longitude of $81^{\circ} 63^{\prime} \mathrm{E}$ and altitude of $298.15 \mathrm{~m}$ above mean sea level. Soil was clay in nature with low nitrogen $\left(226.7 \mathrm{~kg} \mathrm{ha}^{-1}\right)$ medium phosphorus $(13.8 \mathrm{~kg}$ $\mathrm{ha}^{-1}$ ) and high potassium (362.0 $\left.\mathrm{kg} \mathrm{ha}^{-1}\right)$ level having neutral $\mathrm{pH}$. The treatments were $\mathrm{T}_{1^{-}}$ BAS 835 UBH (Imazethapyr 23g/l + Imazamox $23 \mathrm{~g} / \mathrm{l}+$ Bentazone $460 \mathrm{~g} / \mathrm{l})$ @ 759 g a. ha ${ }^{-1}, \mathrm{~T}_{2^{-}}$BAS 835 UBH (Imazethapyr 23g/l + Imazamox $23 \mathrm{~g} / \mathrm{l}+$ Bentazone $460 \mathrm{~g} / \mathrm{l}$ ) (a) $1518 \mathrm{~g}$ a. ha ${ }^{-1}, \mathrm{~T}_{3}$-BAS $835 \mathrm{UBH}$ (Imazethapyr 23g/l + Imazamox $23 \mathrm{~g} / 1+$ Bentazone $460 \mathrm{~g} / \mathrm{l}$ ) @ $800 \mathrm{~g}$ a. ha ${ }^{-1}, \mathrm{~T}_{4^{-}}$ Bentazone 48\% SL @ 960 g a. ha ${ }^{-1}, \mathrm{~T}_{5^{-}}$ Imazethapre 10\% SL @ $100 \mathrm{~g}$ a.i. ha ${ }^{-1}, \mathrm{~T}_{6^{-}}$ Imazamox 12\% SL @ $42 \mathrm{~g}$ a. ha ${ }^{-1}, \mathrm{~T}_{7^{-}}$
Chlorimuron ethyl 25\% WP @ 9 g a.i. ha ${ }^{-1}$, $\mathrm{T}_{8}$-Hand weeding at 20 and 40 DAS and $\mathrm{T}_{9^{-}}$ Untreated check. At 14 DAS, as early post emergence all herbicides were applied. Recommended dose of 25:60:40 kg N: $\mathrm{P}_{2} \mathrm{O}_{5}$ : $\mathrm{K}_{2} \mathrm{O}$ ha $^{-1}$ were applied through Urea, Single Super Phosphate and Murate of Potash as basal in rows uniformly to each plot. As a test crop 'JS 97-52 variety of soybean was sown. The crop was sown in July and first week of November harvested. Weed data on total density and dry weight of weeds were examined at pre spray, after 30, 60 days of spraying and at time of harvest using $1.0 \mathrm{~m}^{2}$ quadrate at one place in a plot. While observations on grain yield and yield attributing parameters viz., no. of branches plant $^{-1}$, pods plant ${ }^{-1}$, seeds pod ${ }^{-1}$, seed index were noticed at time of harvest.

\section{Results and Discussion}

\section{Weed flora}

The weed flora Echinochloa colonum, Cynodon dactylon, Alternanthera sessilis, Cyperus rotundus,Euphorbia geniculata, Digitaria sanguinalis, Phyllathus niruri, Eragrostis pilosa and Commelina benghalensis were recorded at pre spray,after 30, 60 days of spraying and at time of harvest. Broad leaved like Alternanthera sessilis and narrow leaved like, Cynodon dactylon, Echinochloa colonum and Cyperus rotundus weed species were dominant in soybean experiment field.

\section{Effect on weeds}

All the weed-control treatments greatly reduced the individual population of weeds and their total dry weight against untreated check. A combination of reduced dose of bentazone with other herbicides like some imidazolinone herbicides application may reduce cost and improves weed control in soybean (Devlin et al., 1991). Untreated check 
resulted significantly highest density of weed species namely Echinochloa colonum, Cynodon dactylon, Alternanthera sessilis, Commelina benghalensis, Euphorbia geniculata, Digitaria sanguinalis, Phyllanthus niruri, Cyperus rotundus, after 30, 60 days of spraying and at time of harvest, followed by treatment, Chlorimuron ethyl 25\%WP @9 g a. ha ${ }^{-1}\left(\mathrm{~T}_{7}\right)$, highest density and total dry weight of weeds as compared to other herbicide treatments. Significantly lowest density of all the weed species were noticed under treatment hand weeding twice at 20 and 40 DAS $\left(\mathrm{T}_{8}\right)$, throughout the investigation period. This was because of not any weed management practices was carried to control weeds, so weeds proliferates and competition increases with crop for available moisture, nutrient and sunlight that reduces crop yield. Similar findings suggested by Prabhakaran et al., (1992) and Chavan et al., (1990).

As far as dry matter production of total species and other species of weeds examined, significantly highest weed dry matter noticed under untreated check $\left(\mathrm{T}_{9}\right)$ and significantly lowest dry matter production under treatment hand weeding twice at 20 DAS and 40 DAS $\left(\mathrm{T}_{8}\right)$, throughout the investigation period. Similar findings noticed by Patra (1987) (Table 1).

Weed species namely Echinochloa colonum, Cynodon dactylon, Alternanthera sessilis, Cyperus spp., Euphorbia geniculata and Celosia argentea produced significantly maximum dry matter under untreated check $\left(\mathrm{T}_{9}\right)$. Significantly lowest dry matter production under treatment hand weeding twice at 20 DAS and 40 DAS $\left(T_{8}\right)$, throughout the investigation period.

Weed control efficiency based on weed biomass numerically highest under treatment hand weeding twice at 20 DAS and 40 DAS $\left(\mathrm{T}_{8}\right)$ followed Imazethapyr 10\% SL@ $100 \mathrm{~g}$ a.i. ha ${ }^{-1}$ and Imazamox @ 42 g a.i. ha ${ }^{-1}$.
Similar results were noticed by Rajput and Kushwah (2004).

Among the herbicide mixture applied, maximum weed control efficiency was observed under treatment $\left(\mathrm{T}_{3}\right)$ BAS $835 \mathrm{UBH}$ (Imazethapyr $23 \mathrm{~g} / 1$ + Imazamox $23 \mathrm{~g} / \mathrm{l}$ + Bentazone $460 \mathrm{~g} / \mathrm{l}$ ) @ $800 \mathrm{~g}$ a.i.ha ${ }^{-1}$ against other mixture.

Weed index significantly influenced by weed management practices. Highest weed index was examined under untreated check $(62.15 \%)$, whereas lowest weed index was found (2.93) under Imazethapyr @ $100 \mathrm{~g}$ a.i. $\mathrm{ha}^{-1}$ followed by Imazamox @ $42 \mathrm{~g}$ a.i. ha ${ }^{1}(6.57 \%)$. It was found that in mixture herbicide weed index decreases when BAS 835UBH (Imazethapyr $23 \mathrm{~g} / 1$ + Imazamox 23 $\mathrm{g} / \mathrm{l}+$ Bentazone $460 \mathrm{~g} / \mathrm{l}$ ) @ $800 \mathrm{~g}$ a.i.ha ${ }^{-1}$ applied, as compared to other mixture herbicide treatment.

\section{Effect on crop}

Lower weed population and higher weed control efficiency increases grain production. The highest yield was produced by hand weeding twice at 20 and 40 DAS $\left(\mathrm{T}_{8}\right)$ which was comparable with treatment Imazethapyr @ $100 \mathrm{~g}$ a.i. ha ${ }^{-1}$ and Imazamox @ 42 g a.i. $\mathrm{ha}^{-1}$ and mixture herbicide. Significantly lowest yield of seed noticed under weedy check $\left(\mathrm{T}_{9}\right)$. Similar results were reported by Dubey et al., (2000).

The harvest index increases significantly under hand weeding twice at 20 and 40 DAS (52.83) which was comparable with imazethapyr @ 100 g a.i. ha ${ }^{-1}$ (46.28), imazamox @ 42 g a.i. ha ${ }^{-1}$ (44.33).When herbicide were applied in mixture, BAS 835 UBH (imazethapyr $23 \mathrm{~g} / \mathrm{l}+$ imazamox $23 \mathrm{~g} / \mathrm{l}+$ bentazone $460 \mathrm{~g} / \mathrm{l}) @ 800 \mathrm{~g}$ a.i. ha ${ }^{-1}$ registered the highest harvest index (36.53) over other two herbicide mixture doses (Table 2). 
Table.1 Total weed density $\left(\mathrm{m}^{-1}\right)$, Total weed dry matter production $\left(\mathrm{g} \mathrm{m}^{-1}\right)$, WCE $(\%)$, Weed index $(\%)$, Seed yield( $\mathrm{q}$ ha $\left.{ }^{-1}\right)$ and Harvest index $(\%)$

\begin{tabular}{|c|c|c|c|c|c|c|c|c|c|c|c|c|c|}
\hline \multirow[b]{2}{*}{ Weed management practices } & \multicolumn{5}{|c|}{ Total weed density $\left(\mathrm{m}^{-2}\right)$} & \multicolumn{4}{|c|}{ Total weed dry matter production $\left(\mathrm{g} \mathrm{m}^{-1}\right)$} & \multirow{2}{*}{$\begin{array}{l}\text { WCE } \\
(\%) \text { At } \\
\text { harvest }\end{array}$} & \multirow{2}{*}{$\begin{array}{c}\text { Weed } \\
\text { index } \\
(\%)\end{array}$} & \multirow{2}{*}{$\begin{array}{c}\text { Seed } \\
\text { yield } \\
\left(\mathrm{q} \mathrm{ha}^{-1}\right)\end{array}$} & \multirow{2}{*}{$\begin{array}{l}\mathrm{HI} \\
(\%)\end{array}$} \\
\hline & $\begin{array}{c}\text { Time of } \\
\text { application }\end{array}$ & $\begin{array}{c}\text { Pre } \\
\text { spray }\end{array}$ & $\begin{array}{l}\text { 30 day } \\
\text { after } \\
\text { spraying }\end{array}$ & $\begin{array}{l}60 \text { day } \\
\text { after } \\
\text { sprayin } \\
\text { g }\end{array}$ & $\begin{array}{c}\text { At } \\
\text { harvest }\end{array}$ & $\begin{array}{c}\text { Pre } \\
\text { spra } \\
\text { y }\end{array}$ & $\begin{array}{l}\text { 30 day } \\
\text { after } \\
\text { spraying }\end{array}$ & $\begin{array}{c}\mathbf{6 0} \text { day } \\
\text { after } \\
\text { spraying }\end{array}$ & $\begin{array}{c}\text { At } \\
\text { harvest }\end{array}$ & & & & \\
\hline $\begin{array}{l}\text { T}_{1} \text {-BAS 835UBH (Imazethapyr } 23 \\
\text { g/l + Imazamox 23 g/l + } \\
\text { Bentazone } 460 \mathrm{~g} / \mathrm{l}) @ 759 \mathrm{~g} \\
\text { a.i.ha }{ }^{-1}\end{array}$ & 14 DAS & 89.00 & $\begin{array}{c}\mathbf{8 . 7 8} \\
(77.33)\end{array}$ & $\begin{array}{c}\mathbf{6 . 1 7} \\
(37.6)\end{array}$ & $\begin{array}{c}\mathbf{5 . 7 5} \\
(32.6)\end{array}$ & 3.98 & $\begin{array}{c}6.97 \\
(48.1)\end{array}$ & $\begin{array}{c}\mathbf{8 . 1 0} \\
(65.1)\end{array}$ & $\begin{array}{c}8.97 \\
(80.1)\end{array}$ & 69.13 & 26.46 & 16.83 & 31.11 \\
\hline $\begin{array}{l}\text { T }_{2} \text {-BAS 835UBH (Imazethapyr } 23 \\
\text { g/l + Imazamox 23 g/l + } \\
\text { Bentazone } 460 \mathrm{~g} / \mathrm{l}) @ 1518 \mathrm{~g} \\
\text { a.i.ha }^{-1}\end{array}$ & 14 DAS & 68.33 & $\begin{array}{c}\mathbf{7 . 8 5} \\
(63.00)\end{array}$ & $\begin{array}{c}\mathbf{5 . 7 7} \\
(33.0)\end{array}$ & $\begin{array}{c}\mathbf{5 . 3 0} \\
(27.6)\end{array}$ & 3.40 & $\begin{array}{c}6.73 \\
(44.8)\end{array}$ & $\begin{array}{c}7.81 \\
(60.7)\end{array}$ & $\begin{array}{c}8.43 \\
(70.7)\end{array}$ & 72.76 & 25.25 & 17.11 & 35.00 \\
\hline $\begin{array}{l}\text { T }_{3} \text {-BAS 835UBH (Imazethapyr } 23 \\
\text { g/l + Imazamox 23 g/l + } \\
\text { Bentazone } 460 \mathrm{~g} / \mathrm{l}) @ 800 \mathrm{~g} \\
\text { a.i.hat }\end{array}$ & 14 DAS & 69.00 & $\begin{array}{c}7.31 \\
(53.00)\end{array}$ & $\begin{array}{c}\mathbf{5 . 6 7} \\
(31.6)\end{array}$ & $\begin{array}{l}4.56 \\
(20.3)\end{array}$ & 3.55 & $\begin{array}{c}6.39 \\
(40.4)\end{array}$ & $\begin{array}{c}7.49 \\
(55.7)\end{array}$ & $\begin{array}{c}7.72 \\
(59.0)\end{array}$ & 77.18 & 23.40 & 17.53 & 36.53 \\
\hline $\begin{array}{l}\mathrm{T}_{4}-\text { Bentazone } 48 \% \mathrm{SL} @ 960 \mathrm{~g} \\
\text { a.i.ha-1 }\end{array}$ & 14 DAS & 80.67 & $\begin{array}{c}\mathbf{7 . 1 8} \\
(51.3)\end{array}$ & $\begin{array}{c}7.09 \\
(50.0)\end{array}$ & $\begin{array}{c}\mathbf{5 . 3 9} \\
(28.6)\end{array}$ & 3.28 & $\begin{array}{c}\mathbf{5 . 8 2} \\
(33.5)\end{array}$ & $\begin{array}{c}\mathbf{6 . 8 6} \\
(46.6)\end{array}$ & $\begin{array}{c}7.33 \\
(53.2)\end{array}$ & 79.44 & 19.54 & 18.42 & 41.19 \\
\hline $\begin{array}{l}\mathrm{T}_{5^{-}} \text {Imazethapyr 10\% SL @ } 100 \mathrm{~g} \\
\text { a.i.ha }^{-1}\end{array}$ & 14 DAS & 50.00 & $\begin{array}{c}\mathbf{5 . 3 7} \\
(28.33)\end{array}$ & $\begin{array}{l}\mathbf{4 . 7 6} \\
(22.3)\end{array}$ & $\begin{array}{c}3.13 \\
(9.33)\end{array}$ & 1.94 & $\begin{array}{l}4.55 \\
(20.3)\end{array}$ & $\begin{array}{c}4.75 \\
(22.9)\end{array}$ & $\begin{array}{c}\mathbf{5 . 0 4} \\
(25.1)\end{array}$ & 90.20 & 2.93 & 22.22 & 46.28 \\
\hline $\begin{array}{l}\mathrm{T}_{6}-\operatorname{Imazamox}_{\text {a.i.ha }^{-1}} \text { 12\% SL @ } 42 \mathrm{~g} \\
\text { a }\end{array}$ & 14 DAS & 66.67 & $\begin{array}{c}\mathbf{6 . 1 2} \\
(37.3)\end{array}$ & $\begin{array}{c}6.39 \\
(41.0)\end{array}$ & $\begin{array}{c}4.94 \\
(24.0)\end{array}$ & 6.34 & $\begin{array}{c}\mathbf{5 . 2 8} \\
(27.8)\end{array}$ & $\begin{array}{c}6.97 \\
(48.5)\end{array}$ & $\begin{array}{c}7.19 \\
(51.3)\end{array}$ & 80.16 & 6.57 & 21.39 & 44.33 \\
\hline $\begin{array}{l}\mathrm{T}_{7-} \text { Chlorimuron Ethyl 25\% WP } \\
\text { @ } 9 \mathrm{~g} \text { a.i.ha }^{-1}\end{array}$ & 14 DAS & 97.33 & $\begin{array}{c}\mathbf{1 0 . 1 5} \\
(103.0)\end{array}$ & $\begin{array}{c}7.40 \\
(55.6)\end{array}$ & $\begin{array}{c}6.28 \\
(39.0)\end{array}$ & 5.05 & $\begin{array}{c}8.05 \\
(64.4)\end{array}$ & $\begin{array}{c}\mathbf{8 . 5 2} \\
(72.1)\end{array}$ & $\begin{array}{c}9.80 \\
(95.6)\end{array}$ & 63.03 & 36.17 & 14.61 & 26.11 \\
\hline $\begin{array}{l}T_{8} \text { - Hand weeding (twice } 20 \& 40 \\
\text { DAS) }\end{array}$ & $\begin{array}{l}20 \& 40 \\
\text { DAS }\end{array}$ & 82.33 & $\begin{array}{c}\mathbf{1 . 2 5} \\
(1.67)\end{array}$ & $\begin{array}{c}4.99 \\
(24.6)\end{array}$ & $\begin{array}{c}3.74 \\
(13.6)\end{array}$ & 5.82 & $\begin{array}{l}\mathbf{1 . 2 0} \\
(1.4)\end{array}$ & $\begin{array}{c}\mathbf{3 . 3 2} \\
(10.7)\end{array}$ & $\begin{array}{c}\mathbf{3 . 5 0} \\
(11.8)\end{array}$ & 95.45 & - & 22.89 & 52.83 \\
\hline$T_{9}$ - Untreated check & - & 94.00 & $\begin{array}{c}\mathbf{1 0 . 2 5} \\
(168.3)\end{array}$ & $\begin{array}{c}9.75 \\
(94.6)\end{array}$ & $\begin{array}{c}8.00 \\
(64.0)\end{array}$ & 6.69 & $\begin{array}{c}\mathbf{1 3 . 7 6} \\
(188.8)\end{array}$ & $\begin{array}{l}14.93 \\
(222.5)\end{array}$ & $\begin{array}{c}16.11 \\
(259.2)\end{array}$ & 0.00 & 62.15 & 8.66 & 19.83 \\
\hline $\mathrm{SEm} \pm$ & - & - & 0.46 & 0.34 & 0.18 & - & 0.26 & 0.72 & 0.59 & 0.95 & - & 0.56 & 2.94 \\
\hline $\mathrm{CD}$ & - & - & 1.40 & 1.02 & 0.53 & - & 0.78 & 0.82 & 0.20 & 2.84 & - & 1.67 & 8.81 \\
\hline
\end{tabular}


Table. 2 Economics of soybean as affected by weed management practices

\begin{tabular}{|c|c|c|c|c|c|c|}
\hline \multirow[t]{2}{*}{ Integrated weed management practices } & \multicolumn{3}{|c|}{$\begin{array}{l}\text { Cost of cultivation } \\
\left(\mathrm{Rs} \mathrm{ha}^{-1}\right)\end{array}$} & \multirow[t]{2}{*}{$\begin{array}{l}\text { Gross returns } \\
\quad\left(\mathrm{Rs} \mathrm{ha}^{-1}\right)\end{array}$} & \multirow[t]{2}{*}{$\begin{array}{l}\text { Net returns } \\
\left(\text { Rs ha }^{-1}\right)\end{array}$} & \multirow[t]{2}{*}{$\begin{array}{l}\text { Benefit : Cost } \\
\quad \text { ratio }\end{array}$} \\
\hline & Fixed cost & $\begin{array}{l}\text { Treatment } \\
\text { cost }\end{array}$ & Total cost & & & \\
\hline $\begin{array}{l}\text { T}_{1} \text {-BAS 835UBH (Imazethapyr } 23 \mathrm{~g} / \mathrm{l}+\text { Imazamox } 23 \mathrm{~g} / \mathrm{l}+ \\
\text { Bentazone } 460 \mathrm{~g} / \mathrm{l}) @ 759 \mathrm{~g} \text { a.i.ha }\end{array}$ & 22405 & 1111 & 23516 & 51229 & 27713 & 1.18 \\
\hline $\begin{array}{l}\text { T}_{2} \text {-BAS 835UBH (Imazethapyr } 23 \mathrm{~g} / \mathrm{l}+\text { Imazamox } 23 \mathrm{~g} / \mathrm{l}+ \\
\text { Bentazone } 460 \mathrm{~g} / \mathrm{l}) \text { @ } 1518 \mathrm{~g} \text { a.i.ha }\end{array}$ & 22405 & 2022 & 24427 & 52580 & 28153 & 1.15 \\
\hline $\begin{array}{l}\text { T }_{3} \text {-BAS 835UBH (Imazethapyr } 23 \mathrm{~g} / \mathrm{l}+\text { Imazamox } 23 \mathrm{~g} / \mathrm{l}+ \\
\text { Bentazone } 460 \mathrm{~g} / \mathrm{l}) @ 800 \mathrm{~g} \text { a.i.ha }\end{array}$ & 22405 & 1160 & 23565 & 53984 & 30418 & 1.29 \\
\hline $\mathrm{T}_{4}-$ Bentazone 48\% SL @ $960 \mathrm{~g}$ a.i.ha ${ }^{-1}$ & 22405 & 776 & 23181 & 57135 & 33954 & 1.46 \\
\hline $\mathrm{T}_{5}$ - Imazethapyr 10\% SL @ $100 \mathrm{~g}$ a.i.ha ${ }^{-1}$ & 22405 & 305 & 22710 & 68452 & 45741 & 2.01 \\
\hline T6- Imazamox 12\% SL @ 42 g a.i.ha $^{-1}$ & 22405 & 259 & 22664 & 65848 & 43183 & 1.91 \\
\hline $\mathrm{T}_{7^{-}}$Chlorimuron Ethyl 25\% WP @9 g a.i.ha ${ }^{-1}$ & 22405 & 251 & 22656 & 44309 & 21653 & 0.96 \\
\hline 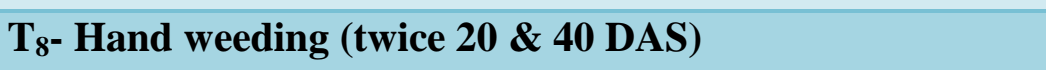 & 22405 & 6000 & 28405 & 71294 & 42889 & 1.51 \\
\hline $\mathbf{T}_{9-}$-Untreated check & 22405 & - & 22405 & 26866 & 4460 & 0.20 \\
\hline
\end{tabular}




\section{References}

Chavan, S.R., Bhorse, R.H., Tumbare, A.D. and Pawar, V.S. (1990). Efficiency of different herbicides in controlling weeds of soybean (Glycine max). Indian Journal of Weed Science. 31(1\&2): 89-90.

Devlin, D.L., Long, J.H., Maddux, L.D. 1991. Using reduced rate of post emergence herbicides in soybeans (Glycine max). Weed Technology, 5, 834-840.

Dubey, M.P., Singh, R., Tiwari, U.K., Kurmavanshi, S.M., Goswami, S.R. and Jain, S.K. (2000). Response of soybean (Glycine max) and associated weeds to pre-emergence application of chlorimuron ethyl and metolachlor. Indian J. Weed Sci., $32(3 \& 4)$ : 153155.

Kumar, M. and Das, T.K. 2008. Integrated weed management for system productivity and economics in soybean (Glycine max) - wheat (Triticum aestivum) system. Indian Journal of Agronomy, 53(3): 189-194.

Patra, A. P. (1987).Weed control in soybean. Indian J. Weed Sci., 19(1 \& 2): 47-51.

Praphakaran, N.R., Kurhania, S.P., Tiwari, J.P. and Jain, K.K. 1992. Weed survey in Kharif oilseeds in soybean crops at Damoh district if Madhaya Pradesh. Abstract Annual Weed Soc. Conf. ISWS/HAU, Hisar, March 3-4,pp.3.

Rajput, R.L. and Kushwah, S.S. (2004). Integrated weed management in soybean on farmers field. Indian $\mathrm{J}$. Weed Sci., 36(3-4): 210-212.

Singh, K.K. and Kharwara, P.C. 1984. Comparative efficacy of some herbicides in controlling weeds in pure stand of soybean. Abstract Annul Weed Science Conference ISWS, Feb. 27-28. PP. 35-36.

\section{How to cite this article:}

Krishna Kumar, Madhu Mali, Shrikant Chitale and Tarun. 2018. Evaluation of Different Levels of New Post Emergence Individual Herbicidal Mixture Over Reference Herbicide in Soybean [Glycine max (L.)]. Int.J.Curr.Microbiol.App.Sci. 7(11): 3167-3172. doi: https://doi.org/10.20546/ijcmas.2018.711.364 